\title{
USO DA LÓGICA FUZZY NA CARACTERIZAÇÃO DO AMBIENTE PRODUTIVO PARA MATRIZES GESTANTES
}

\author{
HÉLITON PANDORFI ${ }^{1}$, IRAN J. O. SILVA ${ }^{2}$, CRISTIANE GUISELINI ${ }^{3}$, \\ SONIA M. S. PIEDADE ${ }^{4}$
}

\begin{abstract}
RESUMO: O objetivo desta pesquisa consistiu na avaliação do ambiente de alojamento, estimando as condições favoráveis ao melhor desempenho de matrizes gestantes. $\mathrm{O}$ experimento foi realizado no período compreendido entre 4-1 e 11-3-2005, em propriedade de produção industrial de suínos, localizada no município de Elias Fausto - SP. A pesquisa foi desenvolvida no setor de gestação, com 24 matrizes primíparas, 12 fêmeas alojadas em baias individuais (T1) e 12 em baias coletivas (T2). O trabalho foi dividido em duas etapas, em função da forma de avaliação dos dados: análise bioclimática e da qualidade do ar, e estimativa dos padrões de conforto térmico ambiental. As variáveis bioclimáticas $\mathrm{T}\left({ }^{\circ} \mathrm{C}\right)$, UR $(\%)$, Tgn $\left({ }^{\circ} \mathrm{C}\right)$ e fisiológicas, taxa respiratória $\left(\operatorname{mov} \min ^{-1}\right)$ e temperatura retal $\left({ }^{\circ} \mathrm{C}\right)$ apontam o sistema de confinamento em baias coletivas como o que possibilitou melhor condicionamento térmico natural às matrizes em gestação. $\mathrm{O}$ uso da teoria dos conjuntos fuzzy permitiu que se fizesse inferência entre os dados resultantes do trabalho experimental com os estabelecidos pela literatura, por intermédio de base de regras, para a determinação do conforto ambiental aplicado a matrizes na fase de gestação.
\end{abstract}

PALAVRAS-CHAVE: conforto térmico, lógica fuzzy, suinocultura.

\section{THE USE OF FUZZY LOGIC FOR THE PRODUCTIVE ENVIRONMENT CHARACTERIZATION FOR PREGNANT SOWS}

\begin{abstract}
The objective of this research was to estimate the environment of housing systems for pregnant sows, as well as variables that have effect on production system. The trial was conducted out from January $4^{\text {th }}$ to March $11^{\text {th }} 2005$ in a specialized farm in industrial production of pork, located in Elias Fausto City, São Paulo State. In gestation facility 24 gilts were allocated: 12 in individual stalls (T1) and 12 in group housing (T2). Basically, this study was divided in two steps in function of the way chosen for data analysis: bioclimatic and air quality analysis; and prediction for environmental thermal comfort patterns. The environmental variables $\left(\mathrm{T},{ }^{\circ} \mathrm{C}\right.$; UR, \%; Tgn, ${ }^{\circ} \mathrm{C}$ ) and physiological (respiratory rate, $\operatorname{mov} \min ^{-1}$; rectal temperature, ${ }^{\circ} \mathrm{C}$ ) indicated that the group housing led to be the better natural thermal environment for pregnant gilts. The fuzzy set theory permitted to compare experimental data with those reported in the literature through rules that establish environment thermal comfort for sows during gestation.
\end{abstract}

KEYWORDS: thermal comfort, fuzzy logic, swine production.

\section{INTRODUÇÃO}

O ambiente de criação intensivo possui influência direta na condição de conforto e bem-estar animal, promovendo a manutenção do balanço térmico no interior das instalações, na qualidade química do ar e na expressão de seus comportamentos naturais, afetando o desempenho produtivo e reprodutivo dos suínos.

\footnotetext{
${ }^{1}$ Eng ${ }^{\mathrm{o}}$ Agrônomo, Prof. Doutor, Departamento de Tecnologia Rural, UFRPE, R. Dom Manoel de Medeiros, s/n, Recife - PE, Fone: (0XX81)3320.6261, pandorfi@ufrpe.br

${ }^{2}$ Eng ${ }^{\circ}$ Agrícola, Prof. Doutor, Departamento de Engenharia Rural, NUPEA - ESALQ/USP, Piracicaba - SP.

${ }^{3}$ Eng $^{\mathrm{O}}$ Agrônomo, Doutor, Física do Ambiente Agrícola, ESALQ/USP, Piracicaba - SP.

${ }^{4}$ Eng ${ }^{0}$ Agrônomo, Prof. Doutor, Departamento de Ciências Exatas, NUPEA - ESALQ/USP, Piracicaba - SP.

Recebido pelo Conselho Editorial em: 23-8-2005
}

Aprovado pelo Conselho Editorial em: 4-12-2006 
BALDWIN (1979) dividiu os componentes ambientais em físicos (temperatura, umidade, ventilação, tipologia das instalações) e sociais (hierarquia, tamanho e composição do grupo, presença ou ausência de animais estranhos) e introduziu o item manejo (dieta, formas de arraçoamento e desmame). $\mathrm{O}$ ambiente físico, por abranger os elementos meteorológicos que afetam os mecanismos de transferência de calor, a regulação e o balanço térmico entre o animal e o meio, exerce forte influência sobre o desempenho e a saúde dos animais (ASHRAE, 1983).

Frente às atuais demandas, não se pode mais considerar sistema de produção animal ou cadeia produtiva, sem considerar o conceito de bem-estar na produção. Diante disso, as utilizações de novas ferramentas da tecnologia da informação favorecem a acurácia das pesquisas e o desenvolvimento de sistemas especialistas para tomada de decisão.

A teoria de conjuntos fuzzy foi introduzida por Lotfi Asker Zadeh, em 1965, como uma teoria matemática aplicada a conceitos difusos. A partir daí, a pesquisa e a aplicação dessa teoria em sistemas de informação têm crescido. Uma área de aplicação da teoria fuzzy é o chamado raciocínio aproximado, semelhante à forma do pensamento humano. Nesses casos, variáveis lingüísticas são representadas por conjuntos fuzzy, interpretando uma variável lingüística como uma variável cujos valores são palavras ou sentenças em linguagem natural, além da existência de variação nas opções (ZADEH, 1965).

Nesse sentido, a introdução dos conhecimentos da teoria dos conjuntos fuzzy mostra-se inovadora. FIRK et al. (2003) relatam melhoria na previsão de inseminação em vacas leiteiras, utilizando a base de dados de identificação de estro, comparado com métodos convencionais. CVETICANIN (2003) apresenta metodologia de pesagem eletrônica para gado, em que um algoritmo foi desenvolvido utilizando a lógica fuzzy. Para o dimensionamento de dietas para gado em condições de semi-estabulação, CADENAS et al. (2003) estipularam um cenário, usando a programação linear fuzzy, associada a processo de tomada de decisão. Essa ferramenta também permitiu a análise da composição das variáveis climáticas para estimar conforto térmico para alojamento de aves poedeiras em produção (OLIVEIRA et al., 1997).

Baseando-se nisso, o objetivo desta pesquisa consistiu na avaliação do ambiente de alojamento, determinando as condições favoráveis ao melhor desempenho animal e na estimativa de padrões de conforto aplicados a matrizes gestantes.

\section{MATERIAL E MÉTODOS}

O experimento foi realizado em uma granja comercial, cuja principal atividade é a produção industrial de suínos, localizada no município de Elias Fausto - SP, apresentando latitude de $22^{\circ} 36^{\prime} \mathrm{S}$, longitude de $47^{\circ} 36^{\prime} \mathrm{W}$ e altitude de $535 \mathrm{~m}$. O clima da região é caracterizado como mesotérmico-Cwa (tropical de altitude), com temperatura do mês mais frio entre 3 e $18{ }^{\circ} \mathrm{C}$, com inverno seco e temperatura do mês mais quente maior que $22{ }^{\circ} \mathrm{C}$, segundo classificação de Köeppen (PEREIRA et al., 2002).

O desenvolvimento da pesquisa foi na fase de gestação, no período compreendido entre 4-1-2005 e 11-3-2005, com duração média de 67 dias. O experimento foi dividido em duas etapas, em função da forma de avaliação dos dados: análise bioclimática e da qualidade do ar nos alojamentos, e estimativa dos padrões de conforto.

Foram avaliados dois sistemas de confinamento, considerados neste trabalho como tratamentos: confinamento em baias individuais de gestação (T1) e confinamento em baias coletivas (T2). Como variáveis-respostas a esses tratamentos, foram consideradas as relações microclimáticas e fisiológicas.

As variáveis meteorológicas foram registradas em intervalos de $15 \mathrm{~min}$, na sala de gestação e no ambiente externo, por meio de sensores instalados à altura de $1,30 \mathrm{~m}$ do piso (instalação), e a 1,50 m em abrigo meteorológico (exterior), conectados a uma plataforma automática de registro de 
dados: temperatura de bulbo seco $\left(\mathrm{Tbs},{ }^{\circ} \mathrm{C}\right)$, umidade relativa do ar (UR, \%) e temperatura de globo negro (Tgn, $\left.{ }^{\circ} \mathrm{C}\right)$.

As concentrações dos gases $\left(\mathrm{O}_{2}, \mathrm{CH}_{4}, \mathrm{NH}_{3}, \mathrm{CO}\right.$ e $\left.\mathrm{H}_{2} \mathrm{~S}\right)$ foram registradas por meio de sensores eletroquímicos, instalados no centro geométrico da instalação, à altura de 1,2 $\mathrm{m}$ do piso, utilizando o equipamento Commander ${ }^{\circledR}$, com detectores calibrados de fábrica com resolução de $1 \mathrm{ppm}$.

A temperatura retal e a taxa respiratória das matrizes foram registradas semanalmente, às $9 \mathrm{~h}$, $12 \mathrm{~h}$ e $17 \mathrm{~h}$. As medidas de temperatura foram tomadas com auxílio de um termômetro digital, Digi Sense, modelo 8522-10, e a taxa respiratória, por observação visual direta e quantificação dos movimentos $\mathrm{min}^{-1}$ por meio de um cronômetro digital Cronobio SW2018. Os animais foram tomados para as avaliações de maneira aleatória, perfazendo amostragem de seis matrizes em cada um dos tratamentos.

A aplicação da lógica fuzzy na avaliação do conforto animal permitiu análise conjunta das variáveis quantitativas de entrada $\left(\mathrm{T},{ }^{\circ} \mathrm{C}\right.$; $\mathrm{UR}, \%$; $\mathrm{NH}_{3}, \mathrm{ppm}$; taxa respiratória, mov $\left.\min ^{-1}\right) \mathrm{e}$ qualitativas de saída (conforto térmico). Para tanto, foi utilizado o Fuzzy Logic Toolbox do MATLAB $^{\circledR} 6.5$ (AMENDOLA et al., 2005).

Dessa forma, foram consideradas as classificações da condição do alojamento em: muito bom (A), bom (B), regular (C) e ruim (D). Em seguida, construiu-se a matriz-demanda (variáveis-respostas) em que a classificação feita anteriormente foi substituída por um critério de pesos, pertencendo ao intervalo de 0 a 1 , em que o valor 1 corresponde a $100 \%$ de veracidade da afirmação. Dessa maneira, foi possível construir uma simulação com maior flexibilidade, visto que os pesos obtidos a partir da literatura podem inferir resultados mais próximos da realidade em relação à sensação de conforto, para as fêmeas em fase de gestação.

Como passo seguinte, foi definida a matriz-oferta, caracterizando as variáveis preditoras (variáveis meteorológicas, concentração de gases e fisiológicas) por termos lingüísticos apropriados (muito alto, alto, médio, baixo e muito baixo) que forneceram a indicação da existência ou da ausência de condição em um ambiente estipulado. A base do desenvolvimento da simulação foi a escolha de um cenário hipoteticamente ideal, com base nas atribuições de pesos, de acordo com dados da literatura, o que permitiu inferir condições de conforto, estresse e suas interfaces.

As variáveis de entrada para os dados meteorológicos constituíram os intervalos de temperatura $\left({ }^{\circ} \mathrm{C}\right)$ e umidade relativa do ar $(\%)$, de acordo com a estimativa das condições de conforto para matrizes gestantes. Todas as classificações foram estipuladas de acordo com as condições limites citadas nos trabalhos de ESMAY (1982); HAHN et al. (1987); PRUNIER et al. (1997), como consta na Tabela 1.

TABELA 1. Classificação do estado de conforto térmico como função das variáveis preditoras temperatura $\left({ }^{\circ} \mathrm{C}\right)$ e umidade relativa do ar $(\%)$.

\begin{tabular}{cccccc}
\hline UR & \multicolumn{5}{c}{ Temperatura $\left({ }^{\circ} \mathrm{C}\right)$} \\
\cline { 2 - 6 }$(\%)$ & $<12$ & $12-21$ & $21-24$ & $24-28$ & $>29$ \\
\hline$<70$ & muito bom $(0,7)$ & muito bom $(1,0)$ & bom $(0,9)$ & regular $(0,7)$ & ruim $(0,8)$ \\
$70-80$ & bom $(0,6)$ & muito bom $(1,0)$ & bom $(0,8)$ & regular $(0,6)$ & ruim $(0,9)$ \\
$>80$ & bom $(0,6)$ & bom $(0,8)$ & regular $(0,8)$ & ruim $(0,6)$ & ruim $(1,0)$ \\
\hline
\end{tabular}

Para as variáveis temperatura $\left({ }^{\circ} \mathrm{C}\right)$ e amônia (ppm), a definição das variáveis lingüísticas caracterizada pelo conforto térmico para matrizes gestantes foi baseada nos resultados encontrados no estudo e de acordo com LARRY et al. (1994), AARNINK \& ELZING (1998), SAMPAIO (2004), conforme apresentado na Tabela 2. 
TABELA 2. Classificação do estado de conforto térmico como função das variáveis preditoras temperatura $\left({ }^{\circ} \mathrm{C}\right)$ e concentração de amônia $\left(\mathrm{NH}_{3}\right)$.

\begin{tabular}{cccccc}
\hline $\mathrm{NH}_{3}$ & \multicolumn{5}{c}{ Temperatura $\left({ }^{\circ} \mathrm{C}\right)$} \\
\cline { 2 - 6 }$(\mathrm{ppm})$ & $<15$ & $15-20$ & $20-25$ & $25-30$ & $>30$ \\
\hline$<1$ & muito bom $(1,0)$ & muito bom $(1,0)$ & bom $(0,8)$ & regular $(0,7)$ & ruim $(0,8)$ \\
$1-5$ & muito bom $(1,0)$ & muito bom $(0,9)$ & bom $(0,7)$ & regular $(0,6)$ & ruim $(0,8)$ \\
$5-10$ & bom $(0,7)$ & bom $(0,8)$ & regular $(0,6)$ & ruim $(0,7)$ & ruim $(1,0)$ \\
$>10$ & regular $(0,5)$ & regular $(0,5)$ & regular $(0,5)$ & ruim $(0,8)$ & ruim $(1,0)$ \\
\hline
\end{tabular}

As variáveis preditoras constituem intervalos de temperatura e da taxa respiratória, definindo a variável-resposta, pela condição de conforto das matrizes. As classificações resultaram da organização da base de dados geradas e apoiadas na literatura (OLIVEIRA et al., 1997; TAVARES et al., 1999; HANNAS et al., 1999), como mostrado na Tabela 3.

TABELA 3. Classificação do estado de conforto térmico como função das variáveis preditoras

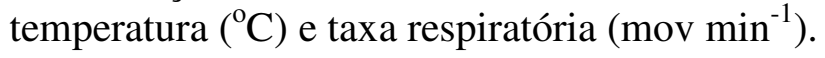

\begin{tabular}{cccccc}
\hline $\begin{array}{c}\text { Taxa respiratória } \\
\left(\text { mov min }^{-1}\right)\end{array}$ & $<15$ & $15-20$ & $20-25$ & $25-30$ & $>30$ \\
\cline { 2 - 6 } & muito bom $(1,0)$ & muito bom $(1,0)$ & bom $(1,0)$ & regular $(0,8)$ & ruim $(0,9)$ \\
$30-50$ & muito bom $(0,8)$ & muito bom $(0,8)$ & bom $(0,8)$ & ruim $(0,8)$ & ruim $(0,9)$ \\
$50-70$ & regular $(0,7)$ & bom $(0,7)$ & regular $(0,6)$ & ruim $(0,9)$ & ruim $(1,0)$ \\
$>70$ & ruim $(0,7)$ & regular $(0,6)$ & ruim $(0,8)$ & ruim $(1,0)$ & ruim $(1,0)$ \\
\hline
\end{tabular}

O método de inferência ou fuzzificação adotado foi o método de Mamdani, que combina os graus de pertinência de cada um dos valores de entrada, por meio do operador mínimo, e agrega as regras pelo operador máximo. Dado um conjunto de valores para a variável-entrada, o sistema obtém um conjunto nebuloso, como o valor da variável de controle. Esse conjunto nebuloso representa uma ordenação de ações de controle aceitáveis naquele momento. Finalmente, uma ação de controle global foi selecionada dentre aquelas aceitáveis, em um processo conhecido como defuzificação (SANDRI \& CORREA, 1999).

As transformações dos resultados fuzzy em valor numérico foram obtidas pela defuzificação, pelo método do centro de área (COA), em que o centro de saída é o centro de gravidade da função de distribuição de possibilidade da ação de controle (MAMDANI, 1976; AMENDOLA et al., 2004).

As análises desenvolvidas neste trabalho poderiam ser implementadas por outros métodos que não a lógica fuzzy, principalmente se forem consideradas apenas as variáveis temperatura, umidade e os usuais índices de conforto térmico. No entanto, a sua implementação pela lógica fuzzy possibilitou expansão que, além de incluir a base de regras de interação entre essas variáveis, permitiu a inclusão de outras variáveis de análise, como concentração de gases e taxa respiratória, visando à determinação do estado de conforto das matrizes suínas.

O delineamento experimental utilizado para a análise geral foi em blocos casualizados (DBC), adotando-se como tratamentos o sistema de confinamento em baias de gestação individuais e coletivas, sendo o número de dias estudados no experimento considerados como blocos. As variáveis meteorológicas, concentração de gases e os parâmetros fisiológicos foram analisados, adotando o DBC para a análise geral, e o teste de Tukey $(\mathrm{p}<0,01)$ para a comparação entre as médias.

\section{RESULTADOS E DISCUSSÃO}

A comparação entre os tratamentos e o ambiente externo, apresentados na Tabela 4, são os resultados médios diários das variáveis-respostas da subdivisão do microambiente estudado (sala 
de gestação), e seus respectivos sistemas de contenção, baias individuais (T1) e baias coletivas (T2). Os tratamentos apresentaram diferenças significativas para temperatura de bulbo seco (Tbs), quando comparados entre si, verificando-se maior temperatura no $\mathrm{T} 1$, em virtude do maior número de animais alojados (animal $\mathrm{m}^{-2}$ ) nesse sistema de contenção, e pelo aporte de energia gerado pelos animais.

Com relação à umidade relativa do ar (UR) e à temperatura de globo negro (Tgn), ambas as variáveis não apresentaram diferenças estatísticas entre tratamentos, no entanto o ambiente externo apresentou a maior média para a UR, caracterizando a condição de entorno à instalação.

TABELA 4. Valores médios diários das variáveis ambientais para o sistema de confinamento em baias individuais (T1), coletivas (T2) e no ambiente externo.

\begin{tabular}{lccc}
\hline & Tbs $\left({ }^{\circ} \mathrm{C}\right)$ & UR $(\%)$ & Tgn $\left({ }^{\circ} \mathrm{C}\right)$ \\
\hline T1 & $25,47 \mathrm{a}$ & $70,48 \mathrm{~b}$ & $25,20 \mathrm{a}$ \\
T2 & $25,03 \mathrm{~b}$ & $70,14 \mathrm{~b}$ & $25,20 \mathrm{a}$ \\
Externo & $24,48 \mathrm{c}$ & $79,65 \mathrm{a}$ & $24,97 \mathrm{a}$ \\
\hline
\end{tabular}

Valores médios com letras diferentes, na mesma coluna, diferem estatisticamente, pelo Teste de Tukey, $\mathrm{p}<0,01$.

As concentrações médias horárias de oxigênio $\left(\mathrm{O}_{2}\right)$ e amônia $\left(\mathrm{NH}_{3}\right)$ na sala de gestação são apresentadas na Tabela 5.

TABELA 5. Concentração média horária dos gases $\mathrm{O}_{2}$ e $\mathrm{NH}_{3}$ no interior da sala de gestação.

\begin{tabular}{crrrrrrrrrrr}
\hline \multirow{2}{*}{ Gases } & \multicolumn{10}{c}{ Horários (h:min) } \\
\cline { 2 - 12 } & \multicolumn{1}{c}{8} & \multicolumn{1}{c}{10} & \multicolumn{1}{c}{10} & \multicolumn{1}{c}{11} & \multicolumn{1}{c}{12} & \multicolumn{1}{c}{13} & \multicolumn{1}{c}{14} & \multicolumn{1}{c}{15} & \multicolumn{1}{c}{16} \\
\hline $\mathrm{O}_{2}(\%)$ & 20,9 & 20,9 & 20,9 & 20,9 & 20,9 & 20,9 & 20,9 & 20,9 & 20,9 & 20,9 & 20,9 \\
$\mathrm{NH}_{3}$ (ppm) & 2,1 & 1,9 & 1,0 & 0,7 & 1,5 & 1,4 & 2,5 & 3,9 & 5,1 & 6,6 & 7,2 \\
\hline
\end{tabular}

Foram encontrados apenas traços de sulfeto de hidrogênio $\left(\mathrm{H}_{2} \mathrm{~S}\right)$, monóxido de carbono $(\mathrm{CO})$ e metano $\left(\mathrm{CH}_{4}\right)$, correspondendo ao limite de resolução do equipamento.

Os valores de oxigênio $\left(\mathrm{O}_{2}\right)$ mantiveram-se dentro das condições recomendadas, verificandose valores estáveis, da ordem de 20,9\%. A presença de oxigênio em nível adequado pode ser considerada indicativo de que não há risco no ambiente quanto à presença dos gases asfixiantes $\left(\mathrm{CH}_{4}\right.$ e $\left.\mathrm{CO}_{2}\right)$, os quais deslocam o oxigênio, conforme descrito por SALIBA \& CORRÊA (2000).

Pode-se verificar que os valores obtidos para concentração de amônia $\left(\mathrm{NH}_{3}\right)$ no interior da instalação foram inferiores para as concentrações admitidas de 10 ppm (BENEDI, 1986), mesmo considerando o horário de pico às $17 \mathrm{~h}$, apresentando valor médio da ordem de 7,2 ppm (LARRY et al., 1994). Atribui-se a baixa concentração de amônia na sala de gestação ao manejo adotado pela granja, que consistia na raspagem diária de dejetos nas baias individuais (período da manhã) e a lavagem das baias coletivas (período da tarde).

A taxa respiratória apresentou diferença estatística entre os tratamentos baias individuais e

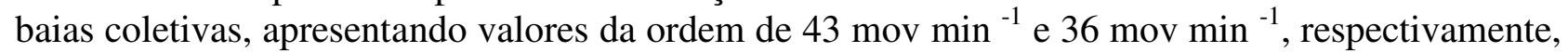
mostrando-se acima da condição normal para animais dessa categoria. Fêmeas gestantes em condição de conforto térmico apresentam taxa respiratória em torno de 15 a 20 mov min ${ }^{-1}$. Os movimentos respiratórios podem aumentar de 22 a 100\%, em função do ambiente térmico, de acordo com HANNAS et al. (1999). Quando a taxa respiratória está elevada, admite-se que a temperatura do ar está próxima à temperatura corporal, o calor é armazenado no organismo e o valor da temperatura corporal aumenta acima dos valores normais, e essa resposta é decorrente do estímulo direto ao centro de calor no hipotálamo, que envia impulso ao sistema cardiorrespiratório, na tentativa de eliminar calor por evaporação por meio da respiração, que, neste caso, apresenta aumento marcante (Tabela 6). 
TABELA 6. Médias das variáveis fisiológicas no período experimental.

\begin{tabular}{lcc}
\hline Tratamentos & Taxa respiratória $\left(\mathrm{mov}^{\mathrm{min}}{ }^{-1}\right)$ & Temperatura retal $\left({ }^{\circ} \mathrm{C}\right)$ \\
\hline Baias individuais & $43 \mathrm{a}$ & $38,91 \mathrm{~b}$ \\
Baias coletivas & $36 \mathrm{~b}$ & $38,72 \mathrm{a}$ \\
\hline
\end{tabular}

Valores médios com letras diferentes, na mesma coluna, diferem estatisticamente, pelo Teste de Tukey, $\mathrm{p}<0,01$.

Com relação à temperatura retal, notam-se diferenças significativas entre os tratamentos, $38,91{ }^{\circ} \mathrm{C}$ (baias individuais) e $38,72{ }^{\circ} \mathrm{C}$ (baias coletivas), porém, para ambos os tratamentos, a variação da temperatura retal encontra-se próxima à condição normal, variando entre 38,6 e $39,3{ }^{\circ} \mathrm{C}$ (Tabela 6). Para matrizes em gestação, o valor considerado ótimo é de $38,6{ }^{\circ} \mathrm{C}$. Mudanças na temperatura retal são observadas em animais submetidos ao estresse por calor, no entanto ocorrem quando o mecanismo de perda de calor evaporativo está sendo insuficiente (MUIRHEAD \& ALEXANDER, 1997).

$\mathrm{Na}$ aplicação da lógica fuzzy para a variável temperatura $\left({ }^{\circ} \mathrm{C}\right)$, considerou-se o domínio do intervalo [7, 40], representando as faixas $<12 ; 12-21 ; 21-24 ; 24-29$ e $>29$, pelos termos lingüísticos: muito baixo, baixo, médio, alto e muito alto, respectivamente (Tabela 1). A variável umidade relativa $(\%)$ denota domínio no intervalo [30,100], representando as faixas $<70 ; 70-80$ e $>80$ pelos termos lingüísticos: baixa, média e alta. As funções de pertinência selecionadas foram trapezoidais, possibilitando o aumento da sensibilidade na construção da base de regras (SANDRI \& CORREA, 1999).

Com base nos dados apresentados neste trabalho, em relação aos limites de conforto ambiental, criou-se um cenário com os termos muito bom (MB), bom (B), regular (RG) e ruim (RM), caracterizando uma base de regras com as definições de pertinência dos termos nebulosos, num domínio [0, 1]. A base de regras foi formada por estruturas do tipo: se <premissa $>$ e $<$ premissa $>$ então <conclusão $>$. Por exemplo: se < umidade relativa é alta $>$ e $<$ temperatura é muito alta $>$ então $<$ conforto térmico é ruim $>$.

Essas regras, juntamente com os dados de entrada foram processados pelo procedimento de inferências, o qual infere a ação de controle de acordo com o conforto térmico dos animais, aplicando o operador de implicação e verificando o grau de compatibilidade global entre os fatos e as cláusulas nas premissas das regras, determinando o valor de conclusão, obtendo-se uma ação de controle geral.

Na Figura 1, observa-se a variação não-linear da condição de conforto térmico, como função da umidade relativa e da temperatura do ar, que foi gerada a partir da base de regras estabelecidas. Dessa forma, verifica-se que os valores de temperatura e umidade apresentam-se como resultado da inferência de um valor, no intervalo $[0,1]$, atribuindo-se o valor 0 para a condição de conforto e 1 para o desconforto térmico das matrizes suínas. Foi possível inferir, para a temperatura de $23,5{ }^{\circ} \mathrm{C} \mathrm{e}$ umidade relativa de $65 \%$, após a defuzificação, o índice de conforto térmico médio de 0,319, mostrado no gráfico de superfície.

Considerando-se os dados médios registrados no interior da instalação, verifica-se que o valor encontrado para temperatura no $\mathrm{T} 1$ foi de $25,47^{\circ} \mathrm{C}$ e umidade relativa do ar de $70,48 \%$ (Tabela 4), permanecendo dentro da faixa limite estabelecida no gráfico gerado, caracterizando-se como condição de conforto (B), não deslocando o índice de 0,319 , a partir da base de regras estabelecidas, após sua verificação.

De maneira análoga, têm-se, para a segunda situação, as variáveis temperatura e concentração de amônia, admitindo-se os termos lingüísticos de acordo com as faixas evidenciadas na Tabela 2. Para a variável temperatura, considerou-se o domínio [10, 40], em que foram atribuídas as denominações: muito baixo, baixo, médio, alto e muito alto. Já para a variável amônia, determinouse o domínio $[0,15]$, com os termos lingüísticos muito baixo, baixo, médio e alto. 


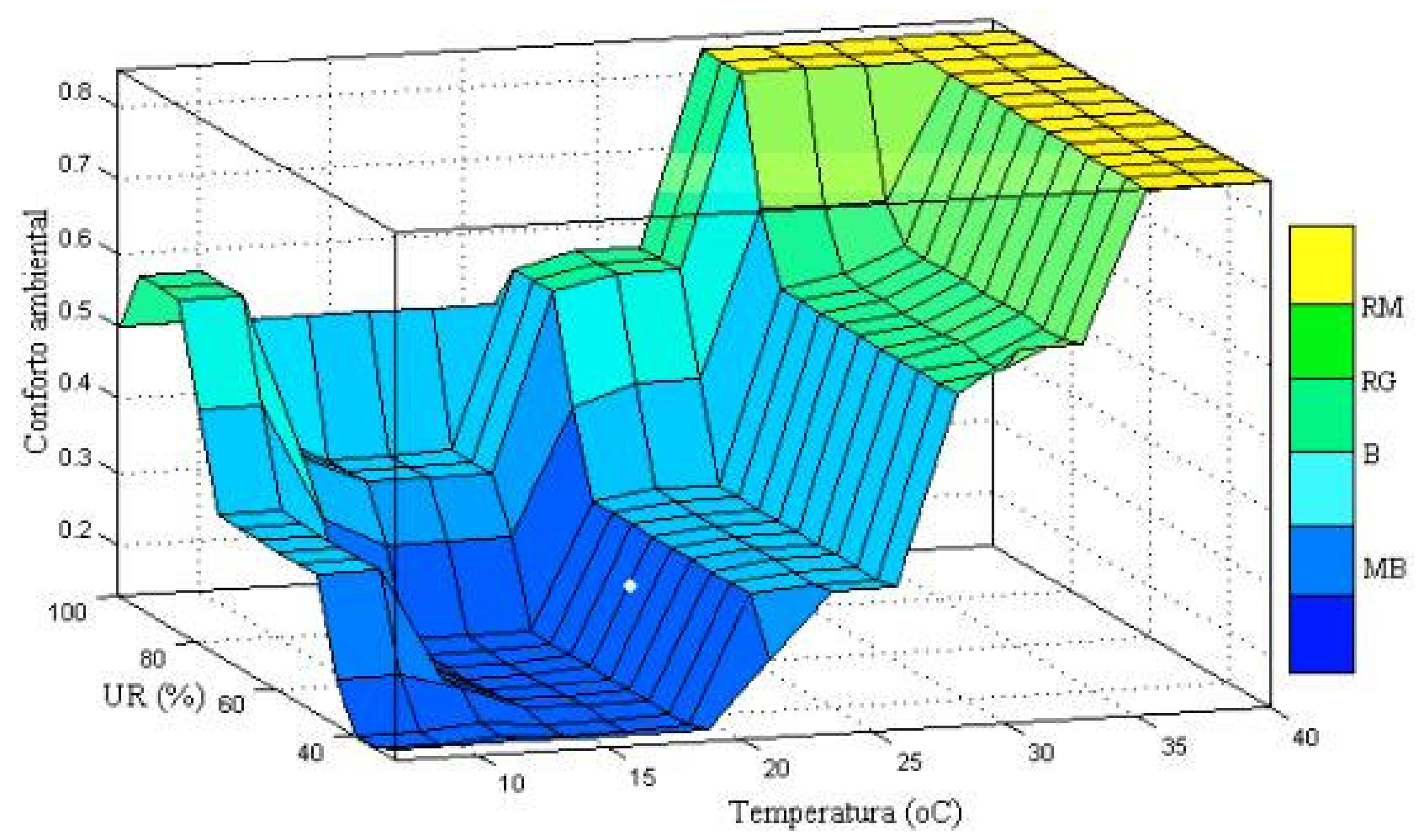

FIGURA 1. Conforto ambiental como função da temperatura e umidade relativa do ar.

Nota-se, na Figura 2, que variação não-linear da variável-resposta conforto térmico, como função das variáveis preditoras concentração de amônia e temperatura do ar, gerada a partir da base de regras, que os valores de temperatura e da concentração de amônia apresentam-se como resultado da inferência de um valor, no intervalo $[0,1]$, representando o conforto dos animais. Com base no gráfico de superfície, foi possível obter inferência para temperatura de $25{ }^{\circ} \mathrm{C}$ e teor de amônia de 7,5 ppm, da condição limite de conforto das fêmeas gestantes, para o índice encontrado de 0,501 .

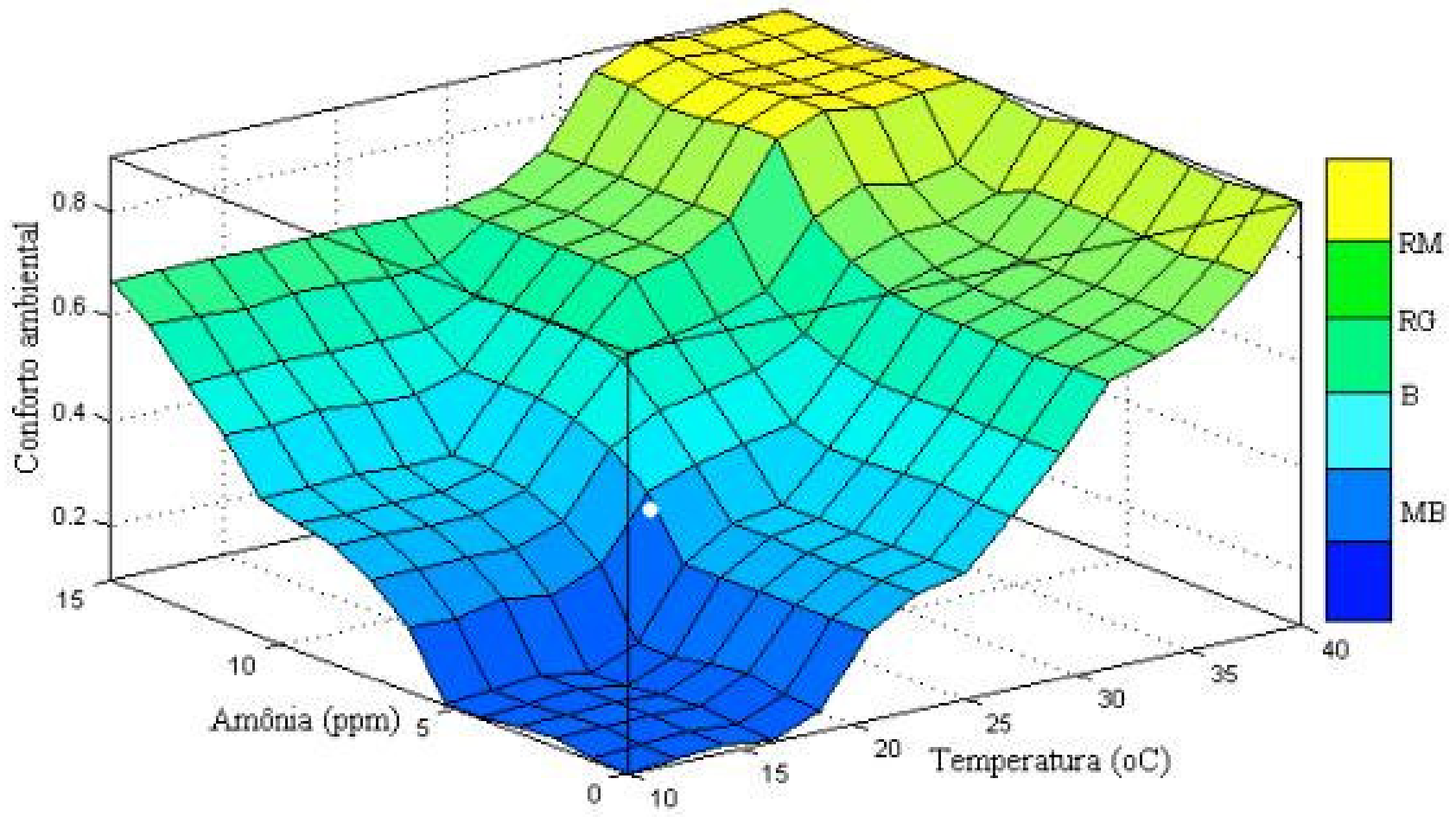

FIGURA 2. Conforto ambiental como função da temperatura e a concentração de amônia. 
Verifica-se, na Tabela 5, que as condições médias para a temperatura $\left({ }^{\circ} \mathrm{C}\right)$ e o teor de amônia (ppm) registrados no interior da edificação apresentaram valores da ordem de 25,47 e 3,3, respectivamente, e, de acordo com a Figura 3, observa-se que, para essas condições, sua classificação, comparativamente com o sistema gerado, encontra-se na faixa $\mathrm{MB}$, a partir do índice de conforto ambiental de 0,336 .

Outra simulação realizada foi para as variáveis independentes, temperatura e taxa respiratória, sendo definidas as variáveis lingüísticas em: muito baixa, baixa, média, alta, muito alta e baixa, média, alta, muito alta, sob o domínio [10, 40] e [20, 80], respectivamente, de acordo com a Tabela 3.

A variável-resposta caracterizada pelo conforto ambiental das matrizes em muito bom, bom, regular e ruim, no intervalo do domínio $[0,1]$, apresentou valores dentro da superfície gerada de $25^{\circ} \mathrm{C}$ para temperatura e de $50 \mathrm{mov} \mathrm{min}^{-1}$ para taxa respiratória, apresentando condição média de conforto de 0,718 , classificado como RG. As classificações foram formuladas com base nos dados registrados, podendo ser alteradas de acordo com o conhecimento de casos específicos localizados, visando à garantia da manutenção do controlador.

Sabendo-se que os valores médios para temperatura e taxa respiratória apresentaram valores de $25,47{ }^{\circ} \mathrm{C}$ e $43 \mathrm{mov} \min ^{-1}$, para a condição mais crítica, com as matrizes alojadas em baias individuais, sua classificação apresenta índice de conforto de 0,691 , ficando abaixo da condição imposta pelo sistema fuzzy, estando entre B e RG.

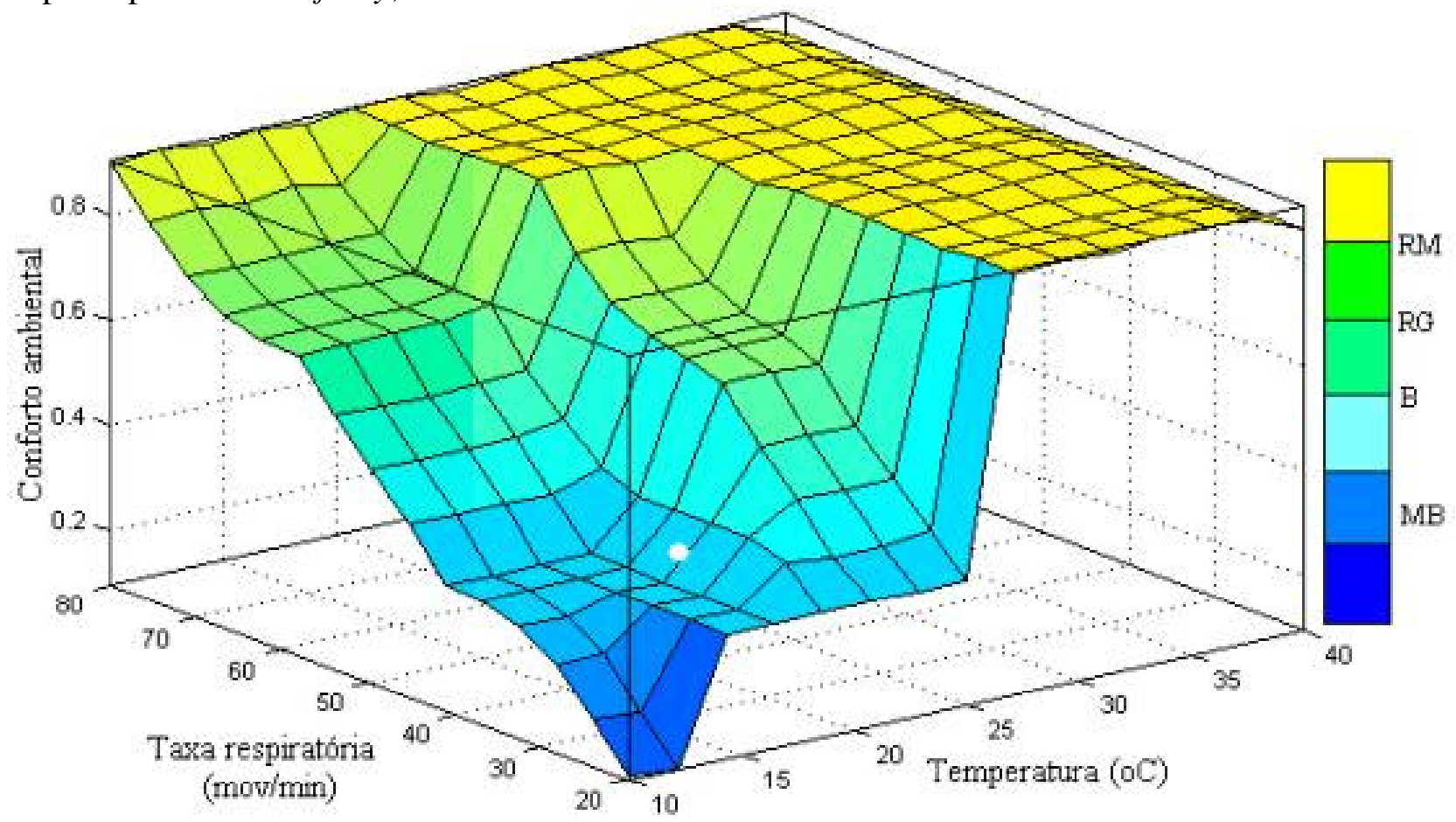

FIGURA 3. Conforto ambiental como função da temperatura e da taxa respiratória.

A utilização da ferramenta permitiu estimar alguns parâmetros ideais de conforto às matrizes gestantes, conforme apresentado na Tabela 7.

TABELA 7. Limite médio do conforto ambiental para matrizes gestantes determinado por meio da teoria dos conjuntos fuzzy $(\alpha)$ e os valores médios registrados no estudo $\left(\alpha^{\prime}\right)$.

\begin{tabular}{lcccc}
\hline Variáveis Preditoras & $\begin{array}{c}\text { Conforto } \\
\text { Ambiental }\end{array}$ & $\begin{array}{c}\text { Valores } \\
\text { Médios }\end{array}$ & $\begin{array}{c}\text { Variável- } \\
\text { Resposta }(\alpha)\end{array}$ & $\begin{array}{c}\text { Variável-Resposta } \\
(\alpha)\end{array}$ \\
\hline Temperatura $\left({ }^{\circ} \mathrm{C}\right)$ & 24,25 & 25,47 & 0,319 & 0,319 \\
Umidade relativa $(\%)$ & 65 & 70,48 & - & - \\
Amônia $(\mathrm{ppm})$ & 10 & 3,3 & 0,501 & 0,336 \\
Taxa respiratória $\left(\mathrm{mov} \mathrm{min}^{-1}\right)$ & 50 & 43 & 0,718 & 0,691 \\
\hline
\end{tabular}


É interessante salientar que a aplicação da lógica fuzzy permitiu estimar os valores médios para os índices de conforto ambiental $(\alpha)$ e, posteriormente, a caracterização das variáveis registradas no ambiente estudado ( $\left.\alpha^{\prime}\right)$, o que possibilitou a comparação entre os índices sugeridos e encontrados no experimento, permitindo inferência em valores intermediários no resultado do ambiente no interior da instalação.

O uso da teoria dos conjuntos fuzzy é interessante e útil para a continuidade de pesquisas desta natureza, pois permite estimativa entre os dados resultantes do trabalho experimental com os estabelecidos pela literatura, por intermédio de base de regras, para a determinação do conforto ambiental aplicado a matrizes na fase de gestação.

\section{CONCLUSÕES}

O sistema de alojamento que se mostrou mais adequado às condições de conforto animal foi o confinamento em baias coletivas, atendendo à demanda por um ambiente que lhe garantiu maior conforto térmico ambiental, potencializando o efeito de sua expressão fisiológica. A utilização da teoria dos conjuntos fuzzy permitiu estabelecer condições de contorno para estimar o conforto das matrizes gestantes.

\section{REFERENCIAS}

AMENDOLA, M.; CASTANHO, M.J.; NÄÄS, I.A.; SOUZA, A.L. Análise matemática de condições de conforto térmico para avicultura usando a teoria dos conjuntos fuzzy. Biomatemática, Campinas, v.14, n.1, p.87-92, 2004.

AMENDOLA, M.; SOUZA, A.L.; BARROS, L.C. Manual do uso da teoria dos conjuntos fuzzy no matlab 6.5. 2005. 46 p. Disponível em: <www.ime.unicamp.br/ laeciocb/MANUAL_2005.pdf/>. Acesso em: 27 maio 2005.

AARNINK, A.J.A.; ELZING, A. Dynamic model for ammonia volatilization in housing with partially slatted floors, for fattening pigs. Livestock Production Science, v.53, n.1, p.153-69, 1998.

ASHRAE. AMERICAN SOCIETY OF HEATING AND REFRIGERATION AND AIR CONDITIONED ENGINEERS. Environment: Handbook of Fundamentals. New York: ASAE, 1983. p.139-98.

BALDWIN, B.A. Operant studies on the behaviour of pigs and sheep in relation to the physical environment. Journal of Animal Science, New Delhi, v.49, n.4, p.1125-7, 1979.

BENEDI, J.M.H. El ambiente de los alojamientos granaderos. Madrid: Ministério da Agricultura, Pesca y Alimentacion, Servicio de Extension Agrária, 1986. 28 p.

CADENAS, J.M.; PELTA, D.A.; PELTA, H.R.; VERDEGAY, J.L. Application of Fuzzy optimization to diet problems in Argentinean farms. European Journal of Operational Research, Amsterdam, v.1, n.5, p.10-12, 2003.

CVETICANIN, D. New approach to the dynamic weighing of livestock. Biosystems Engineering, United Kingdom, v.86, n.2, p.247-52, 2003.

ESMAY, M.L. Principles of animal environment. West Port: ABI, 1982. 325 p.

FIRK, R.; STAMER, E.; JUNGE, W.; KRIETER, J. Improving oestrus detection by combination of activities measurements with information about previous oestrus cases. Livestock Production Sciences, Clare, v.82, n.1, p. 97-103, 2003.

HAHN, G.L.; NIENABER, J.A.; DESHAZER, J.A. Air temperature influences on swine performance and behavior. Applied Engineering in Agriculture, St. Joseph, v.3, n.2, p.295-302, 1987.

HANNAS, M.I.; OLIVEIRA, R.F.M.; DONZELE, J.L.; FERREIRA, A.S.; BARBOSA, R.B.; FERREIRA, R.A.; MORETI, A.M. Efeito da temperatura ambiente sobre os parâmetros 
fisiológicos e hormonais de leitões dos 15 aos $30 \mathrm{~kg}$. In: REUNIÃO ANUAL DA SOCIEDADE BRASILEIRA DE ZOOTECNIA, 36., 1999, Porto Alegre. Anais... Porto Alegre: SBZ, 1999. 1 CD-ROM.

LARRY, D.J.; STEVE, P.; WILLIAM, G.B. Troubleshooting swine ventilation systems: pork industry handbook. Purdue: University Cooperative Extension Service, 1994. 6 p.

MAMDANI, E.H. Advances in the linguistic synthesis of fuzzy controllers. International Journal of Man-Machine Studies, Toronto, v.8, p.669-78, 1976.

MUIRHEAD, M.; ALEXANDER, T. Managing pig health and the treatment of disease. Sheffield: Mentreprises, 1997. 608 p.

OLIVEIRA, R.F.M.; DONZELE, J.L., FREITAS, R.T.F.; FONSECA, F.A. Efeito da temperatura ambiente sobre o desempenho e sobre parâmetros fisiológicos e hormonais de leitões consumindo dietas com diferentes níveis de energia digestível. Revista Brasileira de Zootecnia, Viçosa, v.26, n.3, p.1173-82, 1997.

PEREIRA, A.R.; ANGELOCCI, L.R.; SENTELHAS, P.C. Agrometeorologia: fundamentos e aplicações práticas. Guaíba: Agropecuária, 2002. 478 p.

PRUNIER, A.; MESSIAS DE BRAGANÇA, M.; LE DIVIDICH, J. Influence of high temperature on performance of reproductive sows. Livestock Production Science, Clare, v.52, p.123-33, 1997.

SALIBA, T.M.; CORRÊA, M.A.C. Manual prático de avaliação e controle de gases e vapores. São Paulo: LTR, 2000. 132 p.

SAMPAIO, C.A.P. Caracterização dos sistemas térmicos e acústicos em sistemas de produção de suínos nas fases de creche e terminação. 2004. $121 \mathrm{f}$. Tese (Doutorado em Construções Rurais e Ambiência) - Faculdade de Engenharia Agrícola UNICAMP, Campinas, 2004.

SANDRI, S.; CORREA, C. Lógica nebulosa. In: ESCOLA DE REDES NEURAIS: CONSELHO NACIONAL DE REDES NEURAIS, 5., 1999, São José dos Campos. Anais... São José dos Campos: ITA, 1999. p.73-90.

TAVARES, M.; OLIVEIRA, R.F.M.; DONZELE, J.L.; FERREIRA, A.S. Influência da temperatura ambiente sobre o desempenho e os parâmetros fisiológicos de leitões dos 30 aos $60 \mathrm{~kg}$. Revista Brasileira de Zootecnia, Viçosa, v.28, n.2, 791-8, 1999.

ZADEH, L.A. Fuzzy sets. Information and Control, United Kingdom, v.18, p.338-53, 1965. 